\title{
Об оценках смещения $G$-пика рамановского спектра эпитаксиального графена
}

\author{
() С.Ю. Давыдов \\ Физико-технический институт им. А.Ф. Иофрфе РАН, \\ Санкт-Петербург, Россия \\ Санкт-Петербургский национальный исследовательский университет \\ информационных технологий, механики и оптики, \\ Санкт-Петербург, Россия \\ E-mail: Sergei_Davydov@mail.ru \\ (Поступила в Редакцию 24 мая 2016 г.)
}

В модели двух связанных осцилляторов рассмотрено смещение частоты рамановского пика $G$ эпитаксиального графена вследствие взаимодействия с подложкой, описываемого эффективной силовой константой связи $k$. Показано, что относительный сдвиг $G$-пика $\Delta \omega(G) / \omega(G) \propto k / k_{0 g}$, где $k_{0 g}-$ силовая константа центрального взаимодействия однослойного графена. На основании предположения, что $k \propto P$ и $k \propto-T$ (где $P$ и $T$ - давление и температура) и что именно изменение $k$ является доминирующим, дано качественное объяснение экспериментальных зависимостей $\Delta \omega(G)$ от $P$ и $T$. Кратко обсуждается влияние подложки на уширение $G$-пика эпитаксиального графена.

DOI: 10.21883/FTT.2017.03.44178.209

\section{1. Введение}

Исследования рамановских спектров эпитаксиального графена (ЭГ) интенсивно развиваются [1-3], хотя многие вопросы остаются до сих пор дискуссионными. Так, например, положительный сдвиг $G$-пика ЭГ $\Delta \omega(G)=\Omega(G)-\omega(G)$, где $\Omega(G)(\omega(G))$ - положение $G$-пика в ЭГ (в свободном графене), ${ }^{1}$ во многих работах объясняется механическим напряжением $\sigma$ (точнее, двумерным сжатием), вызванным различием постоянных решеток графена и полупроводниковой подложки (см., например, [4-6]). Впервые зависимость $\Delta \omega(G)$ от $\sigma$ изучалась в работе [7], где рассматривалось влияние статического одноосного напряжения на фононный спектр кремния и было показано, что сдвиг $\Delta \omega(G) \propto \sigma$. В работе [8] этот подход был применен к графитовым волокнам (фибрам). В [9] та же схема рассмотрения была использована для анализа $G$-пика аморфного графитового слоя, сформированного на кремниевой пластине, где внешнее напряжение $\sigma$ вообще отсутствовало. При этом для определения величины положительного сдвига $G$-пика использовался деформационный параметр, полученный экспериментальным путем в [8] для существенно иной системы, что, на наш взгляд, некорректно. Начиная с этой работы апелляция к напряженному (сжатому) состоянию ЭГ при анализе сдвигов $G$-пика стала весьма популярной, при этом практически игнорируется (хотя и упоминается) роль связи слой-подложка, являющейся исходной причиной деформации графенового слоя. Интересно отметить, что в задаче о влиянии субстрата на

\footnotetext{
1 Частота $G$-пика свободного графена $\omega(G)$ соответствует длинноволновым оптическим колебаниям $\omega_{\mathrm{LO}}(\mathbf{k}=0)=\omega_{\mathrm{TO}}(\mathbf{k}=0)$. При этом две подрешетки графена смещаются относительно друг друга (см., например, рис. 9.7 в [1]).
}

электронную структуру ЭГ никогда не рассматривают деформацию как исходную причину изменения закона дисперсии.

Насколько известно автору, первые сомнения в том, что положительный сдвиг $\Delta \omega_{G}$ связан с рассогласованием постоянных решеток графена и субстрата, были высказаны в работе [10] на том основании, что постоянная решетки графена заведомо меньше постоянной решетки субстрата и говорить о сжатом состоянии ЭГ затруднительно. Вместо апелляции к сжатому состоянию в [10] было сделано предположение, что ответственным за сдвиг $\Delta \omega(G)$ является различие линейных коэффициентов расширения графена и подложки. При этом непосредственное взаимодействие графен-подложка вновь лишь упоминалось. По-видимому, впервые это взаимодействие как первопричина изменения рамановского спектра ЭГ было учтено в работе [11], где рассматривался температурный сдвиг $G$-пика в ЭГ (в том числе для ЭГ, сформированного на политипе $6 H-\mathrm{SiC}(0001))$. Подробно и последовательно рамановский и фононный спектры ЭГ, сформированного на поверхности карбида кремния, с учетом буферного слоя экспериментально и теоретически исследованы в [12]. В настоящей работе эффективное взаимодействие слой-подложка учтено модельным образом.

\section{2. Модель двух связанных осцилляторов}

Рассмотрим простейшую модель двух гармонических осцилляторов 1 и 2, имеющих в свободном состоянии собственные частоты $\omega_{1}=\sqrt{k_{1} / M_{1}}$ и $\omega_{2}=\sqrt{k_{2} / M_{2}}$, где $k_{1,2}$ - силовые константы, $M_{1,2}$ - массы атомов. 
Считаем, что эти осцилляторы описывают некоторые характерные колебания слоя и подложки. Пусть теперь между этими системами возникает связь, характеризуемая силовой константой $k$. Тогда можно записать следующие уравнения движения:

$$
\begin{aligned}
& \ddot{x}_{1}=-\omega_{1}^{2} x_{1}-\left(k / M_{1}\right)\left(x_{1}-x_{2}\right), \\
& \ddot{x}_{2}=-\omega_{2}^{2} x_{2}-\left(k / M_{2}\right)\left(x_{2}-x_{1}\right) .
\end{aligned}
$$

Полагая $x_{n}=A_{n} \exp (i \Omega t)$, получим уравнение для определения собственных частот $\Omega$

$$
\left[\left(v_{1}^{2}+\omega_{1}^{2}\right)-\Omega^{2}\right]\left[\left(v_{2}^{2}+\omega_{2}^{2}\right)-\Omega^{2}\right]-v_{1}^{2} v_{2}^{2},
$$

где $v_{1,2}^{2}=k / M_{1,2}$. Общее решение уравнения (2) есть

$$
\begin{aligned}
2 \Omega^{2}= & \left(\omega_{1}^{2}+\omega_{2}^{2}+v_{1}^{2}+v_{2}^{2}\right) \pm\left[\left(\omega_{1}^{2}-\omega_{2}^{2}\right)^{2}\right. \\
& \left.+2\left(v_{1}^{2}-v_{2}^{2}\right)\left(\omega_{1}^{2}-\omega_{2}^{2}\right)+\left(v_{1}^{2}+v_{2}^{2}\right)^{2}\right]^{1 / 2}
\end{aligned}
$$

В приближении слабой связи (другие частные случаи рассмотрены в Приложении), когда выполняются неравенства $v_{1,2}^{2} \ll \min \left\{\omega_{1}^{2}, \omega_{2}^{2}\right\}$ и $\left|\left(v_{1}^{2}-v_{2}^{2}\right) /\left(\omega_{1}^{2}-\omega_{2}^{2}\right)\right| \ll 1$, получим $\Omega_{n} \approx \sqrt{\omega_{n}^{2}+k / M_{n}}$, откуда

$$
\Omega_{n} \approx \omega_{n}\left(1+\frac{k}{2 k_{n}}\right),
$$

где $n=1,2$. Таким образом, сдвиг частоты $\omega_{n}$ из-за взаимодействия равен

$$
\Delta \omega_{n} \equiv \Omega_{n}-\omega_{n} \approx \frac{k}{2 M_{n} \omega_{n}},
$$

а относительный сдвиг

$$
\delta_{n} \equiv \frac{\Delta \omega_{n}}{\omega_{n}} \approx \frac{k}{2 k_{n}} .
$$

По данным работы [4] частота $G$-пика свободного графена $\omega_{g}(G)=1580 \mathrm{~cm}^{-1}$, тогда как для ЭГ на карбиде кремния $\Omega_{g}(G)=1597 \mathrm{~cm}^{-1}$, так что $\Delta \omega_{g}(G)=17 \mathrm{~cm}^{-1}$ и $\delta_{g}(G) \sim 10^{-2}$. Тот же порядок $\delta_{g}(G)$ следует и из данных других указанных здесь работ.

Теперь обратимся к описанию уширения $G$-пика. Будем вновь рассматривать систему двух осцилляторов, но для каждого из них введем собственное затухание $\gamma_{1,2}$ и комплексную силовую константу связи $\tilde{k}=k-i \kappa$. Тогда, добавляя в правую часть системы уравнений (1) слагаемые $-2 \gamma_{1,2} \dot{x}$, получим уравнение для определения затухания связанных осцилляторов $\gamma$ в виде

$$
\left[-2 \gamma \Omega+B_{1}\right]\left[-2 \gamma \Omega+B_{2}\right]-\left(\kappa^{2} / M_{1} M_{2}\right)=0,
$$

где $B_{1,2}=2 \gamma_{1,2} \Omega+\left(\kappa / M_{1,2}\right)$. Будем считать, что $\kappa \ll k, \quad \kappa / M_{1,2} \ll v_{1,2}^{2}$. Полагая для случая слабой связи $(\kappa / \Omega M)^{2} \ll\left(\gamma_{1}-\gamma_{2}\right)^{2}$ (другие частные случаи приведены в Приложении), где приведенная масса $M=2 M_{1} M_{2} /\left(M_{1}+M_{2}\right)$, получим решения вида

$$
\gamma_{1,2}^{*} \approx \gamma_{1,2}+\frac{\kappa}{2 \omega_{g}(G) M_{1,2}},
$$

где мы положили $\Omega=\omega_{g}(G)$.
Полученное выражение аналогично формуле (4): учет взаимодействия приводит к увеличению затухания. Следует подчеркнуть, что сдвиг $G$-пика ЭГ исследован в значительно большей степени, чем его уширение. ${ }^{2}$ Поэтому здесь не будем рассматривать этот вопрос подробно. Можно, однако, отметить работу [4], из которой следует достаточно слабая зависимость ширины пика от связи графенового слоя с $\mathrm{Si}-$ или С-гранью подложки $6 \mathrm{H}-\mathrm{SiC}$.

Отметим, что сделанные в данном разделе оценки логично отнести к квазисвободному графену. Противоположный случай, когда константы связи $k$ и $\kappa$ являются доминирующими параметрами задачи, рассмотрен в Приложении.

\section{3. Оценки константы связи}

В литературе существуют различные модели эпитаксиальных слоев, содержащие наборы силовых констант (см., например, [13-15]). Однако в случае построения таких моделей для ЭГ возникают сложности, связанные с тем, что только часть углеродных атомов связана ковалентным образом с атомами подложки (в случае графена на поверхности $6 H$-SiC эта ситуация хорошо иллюстрируется рис. 3 из работы [4]). Более того, в случае квазисвободного графена связь с подложкой является вандер-ваальсовой. Поэтому под силовыми константами $k$ и $\kappa$ следует понимать модельные константы, отвечающие эффективному взаимодействию графен-подложка.

Мы, однако, сначала обратимся к работе [16], где в рамках модели связывающих орбиталей Харрисона $[17,18]$ показано, что отношение нецентральной силовой константы $k_{1 g}$ к центральной силовой константе $k_{0 g}$ для свободного графена равно 0.22 (см. подробнее [16]). Колебаниям, соответствующим $G$-пику свободного графена, в выражении (6) отвечает силовая константа $k_{n}=k_{0 g}$. С другой стороны, силовую константу $k$ следует считать нецентральной: действительно, по отношению к колебаниям в плоскости ЭГ связь с подложкой соответствует напряжению сдвига. Таким образом, если бы речь шла о двухслойном графене, то отношение $\delta_{g}(G)$ имело бы порядок $10^{-1}$. Поскольку силовые константы $k_{0,1 g}$, согласно [16-18], пропорциональны $d^{-4}, 3$ где $d$ - расстояние между взаимодействующими соседями, для выполнения условия $\delta_{g}(G) \sim 10^{-2}$ достаточно, чтобы отношение расстояния $d_{g}=1.42 \AA$ между соседними

\footnotetext{
${ }^{2}$ Нам во всяком случае не удалось найти количественного анализа дополнительного уширения $G$-пика графена за счет взаимодействия с субстратом.

3 Здесь мы для простоты (имея в виду лишь порядковые оценки) не учитываем вклада коэффициентов гибридизации $[17,18]$ в отношение силовых констант $k / k_{0 g}$. Легко, однако, показать, что при учете связи $p_{z}$-орбитали атома углерода графена с $s p^{3}$-орбиталью атома подложки такого рода поправка дает $d_{\perp} \approx 2.4 \AA$, что практически не отличается от приведенного в тексте результата. Подчеркнем также, что в сделанной оценке используются матричные элементы ковалентной связи. Следует отметить, что, как правило, в модельных работах по двух-, трех- , многослойному или эпитаксиальному графену любое взаимодействие, включая ван-дер-ваальсово, описывается энергией перехода, величину которой можно оценить по формулам Харрисона $[17,18]$ аналогичным образом.
} 
атомами углерода в свободном графене к расстоянию $d_{\perp}$ между графеновым слоем и $\mathrm{SiC}$-подложкой составляло величину $d_{g} / d_{\perp} \sim \sqrt[4]{0.1} \approx 0.56$, откуда $d_{\perp} \approx 2.5 \AA$. Полученный результат представляется вполне разумным, так как значение $d_{\perp}$, во-первых, попадает в интервал между $d_{g}$ и межплоскостным расстоянием $3.35 \AA$ в графите и, во-вторых, удовлетворительно согласуется с экспериментальной оценкой $d_{\perp} \sim 3 \AA$, полученной в [19]. Такие значения $d_{\perp}$ следует приписать квазисвободному графену.

Перейдем теперь к оценкам ангармонических характеристик сдвига $G$-пика ЭГ. Хорошо известно (см., например, работы $[20,21]$ и ссылки, приведенные в них), что в линейном по давлению $P$ приближении произвольную силовую константу $K(P)$ можно представить в виде $K(P)=K(0)+\lambda P$, где $\lambda-$ положительный коэффициент, не зависящий от $P$. Тогда, воспользовавшись выражением (5) и предполагая, что основной эффект давление оказывает на константу связи $k$, для $G$-пика можно записать

$$
\Delta \omega(G) \approx c_{1}+c_{2} P
$$

где $c_{1,2}-$ положительные константы. Экспериментальные результаты изучения влияния давления на рамановский спектр графена представлены в [22,23]. В обеих работах при малых давлениях наблюдалось вполне удовлетворительное согласие с выражением (9). Интересно отметить, что зависимости $\Delta \omega_{g}(G)$ от $P$ с качественной точки зрения одинаковы для однослойного и многослойного графена (включая графит), для одноосного и гидростатического давления, причем для графена, находящегося как в квазисвободном состоянии, так и на подложке $\left(\mathrm{Si} / \mathrm{SiO}_{2}[22]\right)$. Это обстоятельство, на наш взгляд, подтверждает предположение об определяющей роли константы связи графен-подложка $k$ при приложении к ЭГ давления $P$.

Перейдем теперь к обсуждению влияния температуры $T$ на положение $G$-пика ЭГ. Известно (см., например, работы $[20,24]$ и ссылки, приведенные в них), что при высоких температурах (порядка и выше дебаевской) силовые константы можно представить в виде $K(T)=K(0)-\mu T$, где $\mu-$ положительный коэффициент, не зависящий от $T$. Экспериментальные результаты работы [11] действительно показывают близкий к линейному спад частоты $G$-пика с температурой как для квазисвободных графеновых слоев, так и для ЭГ, причем для разных подложек $(6 H-\mathrm{SiC}(0001)$ и $\mathrm{Ni})$. Таким образом, можно записать для $G$-пика

$$
\Delta \omega_{g}(G) \approx c_{3}-c_{4}(T),
$$

где $c_{3,4}$ - положительные константы. Здесь интересно отметить, что для ЭГ на $\mathrm{SiC}$-подложке коэффициент $c_{4}$ в 3 раза больше, чем для квазисвободного графена. Таким образом, как и в случае зависимости от давления, реакция константы связи $k$ на изменение температуры является доминирующей.

\section{4. Заключение}

В работе показано, что сдвиг $G$-пика ЭГ $\Delta \omega(G)$ может быть описан введением эффективной константы взаимодействия графен-подложка $k$. Предположение, что реакция этой константы $k$ на давление и температуру является доминирующей (по сравнению с константами, описывающими изолированные графен и субстрат), позволило качественно объяснить наблюдаемые экспериментально линейные зависимости $\Delta \omega(G)$ от $P$ и $T$. Повторим, что, с нашей точки зрения, описывать сдвиг $G$-пика ЭГ как результат взаимодействия с подложкой более логично, чем апеллировать к таким причинам, как различия постоянных решетки или коэффициентов теплового расширения.

Поскольку частота эпитаксиального графена $\Omega^{2}(G) \propto\left(k_{0 g}+k\right)$, для оценок значения упругих постоянных ЭГ можно использовать модель для однолистного графена (например, модель Китинга [25]), подгоняя силовые константы модели под соответствующие экспериментальные данные.

В заключение обсудим кратко сдвиг $G$-пика в $N$-слойном ЭГ. В настоящее время установлено, что с ростом $N$ $G$-пик смещается в низкочастотную область, стремясь к характерной частоте графита (см., например, рис. 2 в работе [10]). Это легко понять, так как с ростом $N$ влияние подложки, связанной непосредственно только с первым углеродным слоем, на систему, состоящую из $N$ слоев, ослабевает. С точки зрения нашей модели это соответствует уменьшению эффективной константы связи $k$, что и ведет к наблюдаемому эффекту.

Интересно отметить также, что в случае $N$-слойного графена, полученного путем эксфолиации и рассматриваемого как свободный, положение $G$-пика от $N$ не зависит (см. рис. $1, f$ в работе $[3]^{4}$ ). Если применить предложенную нами модель для свободного биграфена $(N=2)$, получим для частот значения (П1). Логично сопоставить частоту $\Omega_{+}\left(\Omega_{-}\right)$антисимметричной (симметричной) моде, когда атомы подрешеток слоев смещаются навстречу друг другу, а колебания в слоях находятся в противофазе (в фазе) [28]. Именно симметричная (раман-активная) мода, частота которой равна $\Omega_{-}=\omega(G)$, определяет положение $G$-пика [2].

Автор признателен В.Ю. Давыдову за привлечение внимания к данной теме.

\section{Приложение}

Из уравнения (3) для одинаковых осцилляторов $\left(\omega_{1}=\omega_{2}=\omega, v_{1}=v_{2}=v\right)$ получаем

$$
\Omega_{+}=\sqrt{\omega^{2}+2 v^{2}}, \quad \Omega_{-}=\omega .
$$

\footnotetext{
${ }^{4}$ Отметим, что в более ранних работах $[26,27]$ сообщалось о зависимости $\omega(G)$ от $N$.
} 
В приближении сильной связи, когда $v_{1,2}^{2} \gg \min \left\{\omega_{1}^{2}, \omega_{2}^{2}\right\}$ и $\left|\left(v_{1}^{2}-v_{2}^{2}\right) /\left(\omega_{1}^{2}-\omega_{2}^{2}\right)\right| \gg 1$, получаем

$$
\begin{gathered}
\Omega_{+} \approx \sqrt{v_{1}^{2}+v_{2}^{2}}\left(1+\frac{\left(\omega_{1} v_{1}\right)^{2}+\left(\omega_{2} v_{2}\right)^{2}}{2\left(v_{1}^{2}+v_{2}^{2}\right)^{2}}\right), \\
\Omega_{-} \approx \sqrt{\frac{\left(\omega_{1} v_{2}\right)^{2}+\left(\omega_{2} v_{1}\right)^{2}}{v_{1}^{2}+v_{2}^{2}}} .
\end{gathered}
$$

Общее решение уравнения (7) имеет вид

$$
\begin{aligned}
\gamma & =\frac{1}{2}\left(\left(\gamma_{1}+\gamma_{2}\right)+\frac{\kappa}{\Omega M}\right) \pm\left[\frac { 1 } { 4 } \left(\left(\gamma_{1}-\gamma_{2}\right)^{2}\right.\right. \\
& \left.\left.+\frac{\kappa^{2}}{\Omega^{2} M^{2}}+2\left(\gamma_{1}+\gamma_{2}\right) \frac{\kappa}{\Omega M}\right)-\frac{1}{2} \frac{\kappa}{\Omega}\left(\frac{\kappa}{M_{2}}+\frac{\gamma_{2}}{M_{1}}\right)\right]^{1 / 2} .
\end{aligned}
$$

Для одинаковых осцилляторов $\left(\gamma_{1}=\gamma_{2}=\gamma, M_{1}=M_{2}=M\right)$ получим

$$
\gamma_{+}=\gamma+\frac{\kappa}{\Omega_{+} M}, \quad \gamma_{-}=\gamma
$$

где $\Omega_{+}$дается выражением (П1). В приближении сильной связи, когда $(\kappa / \Omega M)^{2} \gg\left(\gamma_{1}-\gamma_{2}\right)^{2}$, найдем

$$
\begin{gathered}
\gamma_{+} \approx \frac{\kappa}{\Omega_{+} M}+\frac{1}{2}\left(\gamma_{1}+\gamma_{2}\right)\left(1-\frac{M_{1}-M_{2}}{M_{1}+M_{2}} \frac{\gamma_{1}-\gamma_{2}}{\gamma_{1}+\gamma_{2}}\right), \\
\gamma_{-} \approx \frac{1}{2}\left(\gamma_{1}+\gamma_{2}\right)\left(1+\frac{M_{1}-M_{2}}{M_{1}+M_{2}} \frac{\gamma_{1}-\gamma_{2}}{\gamma_{1}+\gamma_{2}}\right),
\end{gathered}
$$

где $\Omega_{+}$определяется формулой (П2) в случае, если $v_{1,2}^{2} \gg \min \left\{\omega_{1}^{2}, \omega_{2}^{2}\right\}$.

\section{Список литературы}

[1] D. Yoon, H. Cheong. In: Raman spectroscopy for nanomaterials characterization / Ed. by Challa S.S.R. Kumar. Springer (2012). P. 191.

[2] L.M. Malard, M.A. Pimenta, G. Dresselhaus, M.S. Dresselhaus. Phys. Rep. 473, 51 (2009).

[3] A.C. Ferrari, D.M. Basko. Nature Nanotechnol. 8, 235 (2013).

[4] Z.H. Ni, W. Chen, X.F. Fan, J.L. Kuo, T. Yu, A.T.S. Wee, Z.X. Shen. Phys. Rev. B 77, 115416 (2008).

[5] N. Ferralis, R. Maboudian, C. Carraro. Phys. Rev. Lett. 101, 156801 (2008).

[6] J. Robinson, C. Puls, N. Staley, J. Stitt, M. Fanton, K. Emtsev, T. Seyller, Y. Liu. Nano Lett. 9, 964 (2009).

[7] E. Anastassakis, A. Pinczuk, E. Burstein, F.H. Pollak, M. Cardona. Solid State Commun. 8, 133 (1970).

[8] H. Sakata, G. Dresselhaus, M.S. Dresselhaus, M. Enda. J. Appl. Phys. 63, 2769 (1988).

[9] J.W. Ager III, S. Anders, A. Anders, I.G. Brown. Appl. Phys. Lett. 66, 3444 (1995).

[10] J. Röhrl, M. Hundhausen, K. V. Emtsev, T. Seyller, R. Graupner, L. Ley. Appl. Phys. Lett. 92, 201918 (2008).

[11] N. Ferralis, R. Maboudian, C. Carraro, Phys. Rev. B 83, 081410 (2011).

[12] F. Fromm, M.H. Oliveira Jr., A. Molina-Sanchez, M. Hundhausen, J.M. Lopes, H. Riechert, L. Wirtz, T. Seyller. New J. Phys. 15, 043031 (2013).
[13] N.S. Luo, P. Ruggerone, J.P. Tonnies. Phys. Rev. B 54, 5051 (1996).

[14] C. Oshima, A. Nagashima. J. Phys.: Condens. Matter 9, 1 (1997).

[15] A. Politano. arXiv: 1601.00573.

[16] С.Ю. Давыдов. ФТТ 52, 172 (2010).

[17] У. Харрисон. Электронная структура и свойства твердых тел. Мир, М. (1983). Т. 1. 382 с.

[18] С.Ю. Давыдов, О.В. Посредник. Метод связывающих орбиталей в теории полупроводников. Изд-во СПбГЭТУ „ЛЭТИ“, СПб. (2007); twirpx.com/file/1014608/

[19] J. Borysiuk, J. Sołtys, R. Bozek, J. Piechota, S. Krukowski, W. Strupinski, J.M. Baranowski, R. Stepniewski. Phys. Rev. B 85, 045426 (2012).

[20] А.А. Блистанов, В.С. Бондаренко, Н.В. Переломова, Ф.Н. Стрижевская, В.В. Чкалова, М.П. Шаскольская. Акустические кристаллы. Справочник. Наука, М. (1982). $632 \mathrm{c}$

[21] В.М. Грабов, С.Ю. Давыдов, Ю.П. Миронов, А.М. Джумиго. ФТТ 27, 2017 (1985).

[22] J.E. Proctor, E. Gregoryanz, K.S. Novoselov, M. Lotya, J.N. Coleman, M.P. Halsall. Phys. Rev. B 80, 073408 (2009).

[23] S. Lu, M. Yao, X. Yang, Q. Li, J. Xiao, Z. Yao, L. Jiang, R. Liu, Bo Liu, S. Chen, B. Zou, T. Cui, B. Liu. Chem. Phys. Lett. 585, 101 (2013).

[24] С.П. Никаноров, Б.К. Кардашев. Упругость и дислокационная неупругость кристаллов. Наука, М. (1985). 250 с.

[25] С.Ю. Давыдов. ФТТ 52, 756 (2010).

[26] A.C. Ferrari, J.C. Meyer, V. Scardaci, C. Casiraghi, M. Lazzeri, F. Mauri, S. Piscanec, D. Jiang, K.S. Novoselov, S. Roth, A.K. Geim. Phys. Rev. Lett. 97, 187401 (2006).

[27] D. Graf, F. Molitor, K. Ensslin, C. Stampfer, A. Jungen, C. Hierold, L. Wirtz. Nano Lett. 7, 238 (2007).

[28] A. Cocemasov, D. Nika. In: ISPC „Modern information and electronic technologies“. Odessa (2013). P. 130. 